\title{
Corrections to scaling in the forest-fire model
}

\author{
Romualdo Pastor-Satorras and Alessandro Vespignani \\ The Abdus Salam International Centre for Theoretical Physics (ICTP) \\ Condensed Matter Section \\ P.O. Box 586, 34100 Trieste, Italy
}

(June 17, 2018)

\begin{abstract}
We present a systematic study of corrections to scaling in the self-organized critical forest-fire model. The analysis of the steady-state condition for the density of trees allows us to pinpoint the presence of these corrections, which take the form of subdominant exponents modifying the standard finite-size scaling form. Applying an extended version of the moment analysis technique, we find the scaling region of the model and compute the first non-trivial corrections to scaling.

PACS numbers: 05.65.+b, 05.70.Ln
\end{abstract}

\section{INTRODUCTION}

The term self-organized criticality (SOC) [1] refers to a set of driven dissipative systems that, under the action of a very small external driving, evolve into a critical state characterized by avalanches broadly distributed in space and time, which lead to divergent (power-law) response functions. Since its introduction by Bak, Tang, and Wiesenfeld [2], the concept of SOC has been the object of a very intense research activity, covering both theory and numerical simulations.

Among the many models proposed so far exhibiting SOC behavior, the forest-fire model (FFM) [3 6] is one of the most simply defined and well understood. The FFM is a three states cellular automaton defined on a $d$ dimensional hypercubic lattice. Each site of the lattice is occupied either by a tree, by a burning tree, or is empty. Every time step, the cellular automaton evolves according to the following set of rules: (i) each burning tree becomes an empty site; (ii) every tree with at least one burning nearest neighbor becomes a burning tree; (iii) a tree becomes a burning tree with probability $f$, irrespective of its nearest neighbors; (iv) an empty site becomes a tree with probability $p$. The FFM possesses two characteristic time scales [4]: the average time for a tree to grow $1 / p$ and the average time between fires $1 / f$. In the limit of the double infinite time scale separation, $1 \gg p \gg f$, the model displays critical behavior 44.67: i.e., fires are distributed according to power laws. The magnitudes characterizing a fire are the total number of trees burnt $s$, and the total time duration of the fire $t$ (measured as the total number of parallel updatings of the algorithm). In the critical state, with $p / f \gg 1$, the probability distributions of sizes and times have been observed to follow the standard finite-size scaling (FSS) hypothesis [7]:

$$
\begin{aligned}
& P(s, \theta)=s^{-\tau_{s}} \mathcal{F}\left(\frac{s}{\theta^{\lambda_{s}}}\right), \\
& P(t, \theta)=t^{-\tau_{t}} \mathcal{G}\left(\frac{t}{\theta^{\lambda_{t}}}\right),
\end{aligned}
$$

where $\theta=p / f$ is the critical parameter of the model [8], and $\tau_{x}$ and $\lambda_{x}$ are scaling exponents characterizing the critical state [9]. Finally, $\mathcal{F}(z)$ and $\mathcal{G}(z)$ are cut-off functions that are constant for $z \rightarrow 0$ and decay exponentially fast for $z \rightarrow \infty$.

The precise determination of critical exponents is a relevant issue in order to firmly establish universality classes and the upper critical dimension, that on their turn are fundamental in the theoretical understanding of the critical nature of the model. While the numerical determination of the overall power law behavior is a relatively easy task, a very accurate determination of critical exponents from numerical simulations can suffer from strong systematic biases due to the distribution's lower and upper cut-offs. More subtly, the assumption of the FSS form does not take into account the presence of corrections to scaling due to subdominant exponents. These corrections are more evident for small values of the various magnitudes and for deviations from pure criticality $\left(\theta^{-1} \neq 0\right)$. On the other hand, for a sufficiently large value of $\theta$, one can safely assume that the scaling (11)(2) is essentially correct. Let us then define the scaling regime of the model by the parameter $\theta_{\text {scal }}$, defined such that the single scaling picture is correct for $\theta>\theta_{\text {scal }}$; in principle, $\theta_{\text {scal }}$ is a magnitude which depends on the microscopic details of the model. However, the value of $\theta_{\text {scal }}$ is in general unknown, and when analyzing numerical data, there is no a priori way to ascertain whether the range of $\theta$ at our disposal is large enough.

In this paper, we will show that in the stationary state of the forest fire model, the presence of scaling corrections arises naturally. The analytical inspection of the steady state condition points out the presence of subdominant scaling corrections and calls for an extended scaling framework allowing the evaluation of the scaling regime and the various corrections to scaling present in the model. The proper treatment of scaling corrections permits a more precise estimate of the leading exponents. In order to analyze the occurrence of correction to scaling in a systematic way, we generalize the powerful moment analysis introduced in Refs. [10,11] to a more general scaling form. Within this framework, we are able to estimate the value $\theta_{\text {scal }}$, above which the simple form (11) is meaningful. We thus obtain corrected exponents, and 
the values of the first subdominant exponents.

The paper is organized as follows: In Section II, by analyzing the steady state condition, we show the ineluctable emergence of subdominant corrections to scaling in the FFM. In Sec. III, we review the moment analysis technique, and outline its extension to probability distributions with subdominant terms. Sec. IV provides numerical evidence of our results by means of extensive simulations of the FFM in $d=2$. Finally, our conclusions are summarized in Sec. V.

\section{STATIONARITY CONDITION AND SCALING CORRECTIONS}

The necessity to include corrections to scaling indeed arises naturally in the FFM, by just considering the steady-state condition of the model [4.6. For any value of $\theta$, at large times the FFM sets in a steady state characterized by an average constant density of trees, $\rho_{t}$, and empty sites, $1-\rho_{t}$ (the density difference after and before a fire is negligible, being of order $\theta / L^{2}$ ). The density of trees is known to display the asymptotic behavior at large $\theta$ [6,8]

$$
\rho_{t}=\rho_{t}^{\infty}-a \theta^{-\alpha} .
$$

Computer simulations in $d=2$ provide the values $\rho_{t}^{\infty} \simeq$ 0.408 and $\alpha \simeq 0.5$ [8]. In the steady-state, and for a fixed $\theta$ value, the average number of growing trees, $p\left(1-\rho_{t}\right) L^{d}$, must equal the average number of burnt trees, $f \rho_{t}\langle s\rangle_{\theta} L^{d}$, where $\langle s\rangle_{\theta}$ is the average size of a fire. Therefore, the mean number of trees burnt by a fire is given by

$$
\langle s\rangle_{\theta}=\theta \frac{1-\rho_{t}}{\rho_{t}} .
$$

In the limit $\theta \rightarrow \infty$, the mean tree density reaches its critical value $\rho_{t}^{\infty}$, and we recover the usual relation $\langle s\rangle_{\theta} \sim$ $\theta=p / f$ 河. However, for any finite value of $\theta$, the system is subcritical and $\rho_{t}<\rho_{t}^{\infty}$. Substituting the expression (3) into (4), we obtain

$$
\begin{aligned}
\langle s\rangle_{\theta} & =\theta \times \frac{1-\rho_{t}^{\infty}+a \theta^{-\alpha}}{\rho_{t}^{\infty}-a \theta^{-\alpha}} \\
& \simeq \frac{1-\rho_{t}^{\infty}}{\rho_{t}^{\infty}} \theta+\frac{a}{\left(\rho_{t}^{\infty}\right)^{2}} \theta^{1-\alpha}+\mathcal{O}\left(\theta^{1-2 \alpha}\right) .
\end{aligned}
$$

That is, neglecting corrections of order $\theta^{1-2 \alpha}$ (which is valid since $\alpha \sim 0.5$ ), the form of the average fire size for finite $\theta$ is

$$
\langle s\rangle_{\theta}=C_{1} \theta+C_{2} \theta^{1-\alpha},
$$

where the $C_{k}$ are constants independent of $\theta$. Inspection of Eq. (6) proves that it is impossible to obtain such a $\theta$ dependence for the average avalanche size with an FSS of the form (11). We are therefore forced to admit a more complex scaling form. These corrections to scaling, which on the other hand are well-known in the field of equilibrium and non-equilibrium critical phenomena [12], take the form of subdominant corrections to the leading (infinite $\theta$ ) scaling form of the probability distributions. The most general form of these corrections is

$$
P(x, \theta)=x^{-\tau_{x}} \mathcal{F}\left(\frac{x}{\theta^{\lambda_{x}}}\right)+x^{-\tau_{x}^{*}} \mathcal{F}^{*}\left(\frac{x}{\theta^{\lambda_{x}^{*}}}\right)+\ldots
$$

In the last equation, $\tau_{x}^{*}$ and $\lambda_{x}^{*}$ are subdominant exponents, correcting the infinite $\theta$ behavior, and $\mathcal{F}^{*}(z)$ is a cut-off function that decays faster than $\mathcal{F}(z)$ when $z \rightarrow \infty$. In this way, and for fixed $\theta$, the effects of the corrections are expected to be more noticeable for small values of $x$. The ellipsis denotes other possible corrections, which are of lower order compared to the first one.

In this perspective, a very accurate measurement of critical exponents cannot escape the precise knowledge of the extent of the intermediate region in which scaling corrections are still noticeable. In particular, a method of analysis which takes into account the presence of subdominant exponents is required for a fully consistent analysis of the scaling properties at finite values of $\theta$.

\section{MOMENT ANALYSIS}

The determination of the scaling exponents for the FFM has been performed most often in previous works by a direct measurement of the slope of a log-log plot [4, 6, 8, 13, 14]. This procedure yields the exponent $\tau_{s}$ by means of a straightforward linear regression. The exponent $\lambda_{s}$ is then computed by imposing the constraint (11) for different values of $\theta$, using the previously computed value of $\tau_{s}[6]$.

Even though with this procedure (sometimes supplemented with extrapolations and/or local slope analysis) one can determine the exponents within a $10 \%$ accuracy, its performance is affected by the existence of the upper and lower cutoffs, which render difficult its application. Moreover, any binning performed to smooth the numerical distributions can lead to biases very difficult to assess. In this respect, it is better to use analysis techniques that use the whole set of data (not only the power law regime) and contain explicitly the system-size dependency. In the SOC field, the moment analysis has been introduced by De Menech et al. in the context of the two dimensional Bak-Tang-Wiesenfeld model [10,11] and has been successfully applied to both deterministic and stochastic models 15 18. In the following we introduce the moment analysis and extend the method in order to deal with scaling forms that make explicit the presence of subdominant corrections. 


\section{A. Single scaling form}

In this section, we concentrate in the moment analysis of the fire size, following Refs. 10,11. We start with a distribution fulfilling the scaling form (11). The $q$-th moment of the distribution is defined by $\left\langle s^{q}\right\rangle_{\theta}=$ $\int_{1}^{\infty} s^{q} P(s, \theta) d s$. Inserting the scaling form of $P$ into this expression yields the $\theta$ dependence

$$
\left\langle s^{q}\right\rangle_{\theta}=\theta^{\lambda_{s}\left(q+1-\tau_{s}\right)} \int_{\theta^{-\lambda_{s}}}^{\infty} z^{q-\tau_{s}} \mathcal{F}(z) d z,
$$

where we have used the transformation $z=s / \theta^{\lambda_{s}}$. For large values of $\theta$, and provided that $q>\tau_{s}-1$, the lower limit of the integral in (8) can be replaced by 0 . We then have $\left\langle s^{q}\right\rangle_{\theta} \sim \theta^{\lambda_{s}\left(q+1-\tau_{s}\right)}$. In general, we can write $\left\langle s^{q}\right\rangle_{\theta} \sim \theta^{\sigma_{s}(q)}$, where the exponents $\sigma_{s}(q)$ can be obtained as the slope of a log-log plot of $\left\langle s^{q}\right\rangle_{\theta}$ as a function of $\theta$. Comparing with (8), one has $\left\langle s^{q+1}\right\rangle_{\theta} /\left\langle s^{q}\right\rangle_{\theta} \sim \theta^{\lambda_{s}}$ or $\sigma_{s}(q+1)-\sigma_{s}(q)=\lambda_{s}$, so that the slope of $\sigma_{s}(q)$ as a function of $q$ is the cutoff exponent; i.e. $\lambda_{s}=\partial \sigma_{s}(q) / \partial q$. This is not true for small $q$, because the integral in (8) is dominated by its lower cutoff. In particular, the lower cutoff becomes important for $q \leq \tau_{s}-1$. Once the exponent $\lambda_{s}$ is known, we can estimate $\tau_{s}$ from the scaling relationship $\left(2-\tau_{s}\right) \lambda_{s}=\sigma_{s}(1)$.

The results of the moment analysis must finally be checked by means of a data collapse analysis. The initially assumed FSS hypothesis (11) has to be verified, and must be consistent with the calculated exponents. This can be done by rescaling $s \rightarrow s / \theta^{\lambda_{s}}$ and $P(s, \theta) \rightarrow P(s, \theta) \theta^{\lambda_{s} \tau_{s}}$. Data for different values of $\theta$ must then collapse onto the same universal curve if the FSS hypothesis is to be satisfied. Complete consistency between the methods gives the best collapse with the exponents obtained by the moment analysis.

\section{B. Moment analysis with corrections to scaling}

Let us now develop the formalism of the moment analysis for a distribution with corrections to scaling of the form (7), where we will only keep the first non-trivial correction. By plugging this form into the definition of the $q$-th moment, we obtain

$$
\begin{aligned}
\left\langle s^{q}\right\rangle_{\theta} & =\int_{1}^{\infty} s^{-\tau_{s}+q} \mathcal{F}\left(\frac{s}{\theta^{\lambda_{s}}}\right) d s+\int_{1}^{\infty} s^{-\tau_{s}^{*}+q} \mathcal{F}^{*}\left(\frac{s}{\theta^{\lambda_{s}^{*}}}\right) d s \\
& =\theta^{\lambda_{s}\left(q+1-\tau_{s}\right)} \int_{\theta^{-\lambda_{s}}}^{\infty} z^{-\tau_{s}+q} \mathcal{F}(z) d z \\
& +\theta^{\lambda_{s}^{*}\left(q+1-\tau_{s}^{*}\right)} \int_{\theta^{-\lambda_{s}^{*}}}^{\infty} z^{-\tau_{s}^{*}+q} \mathcal{F}^{*}(z) d z .
\end{aligned}
$$

In the integrals of the previous expression we have explicitly written the dependence on the lower cut-off. For $\theta$ sufficiently large and $q>\max \left(\tau_{s}, \tau_{s}^{*}\right)-1$, the lower limits tend to zero, and thus we expect the integrals to be independent of $\theta$. However, we cannot discard in general a possible dependence on $q$ (through the exponent in the integrand). We have therefore

$$
\left\langle s^{q}\right\rangle_{\theta}=C(q) \theta^{\lambda_{s}\left(q+1-\tau_{s}\right)}+C^{*}(q) \theta^{\lambda_{s}^{*}\left(q+1-\tau_{s}^{*}\right)},
$$

where we have defined the constants (independent of $\theta$ )

$$
\begin{aligned}
C(q) & =\int_{0}^{\infty} z^{-\tau_{s}+q} \mathcal{F}(z) d z \\
C^{*}(q) & =\int_{0}^{\infty} z^{-\tau_{s}^{*}+q} \mathcal{F}^{*}(z) d z .
\end{aligned}
$$

Analysis of the general Equation $(10)$ is extremely difficult, due to the impossibility to separate the two leading behaviors $\theta^{\lambda_{s} q}$ and $\theta^{\lambda_{s}^{*} q}$. In order to achieve further progress, we must somehow simplify relation (10). To do so, we proceed to make an ansatz, whose validity will have to be numerically verified a posteriori. The ansatz consists in assuming the identity

$$
\lambda_{s}=\lambda_{s}^{*},
$$

that is, the cut-off exponents do not suffer from corrections. The physical interpretation of this single cut-off exponent for both the leading and subdominant terms in the size probability distribution is related to the presence of a unique and well-defined divergent characteristic size in the avalanche evolution. Under this assumption, Eq. (10) becomes

$$
\left\langle s^{q}\right\rangle_{\theta}=\theta^{\lambda_{s}(q-1)}\left[C(q) \theta^{\lambda_{s}\left(2-\tau_{s}\right)}+C^{*}(q) \theta^{\lambda_{s}\left(2-\tau_{s}^{*}\right)}\right] .
$$

Specializing this relation to $q=1$ we obtain

$$
\langle s\rangle_{\theta}=\left[C(1) \theta^{\lambda_{s}\left(2-\tau_{s}\right)}+C^{*}(1) \theta^{\lambda_{s}\left(2-\tau_{s}^{*}\right)}\right] .
$$

Comparing now with the expression for the average fire size (first moment), Eq. (6), we can identify $\lambda_{s}\left(2-\tau_{s}\right)=$ 1 and $\lambda_{s}\left(2-\tau_{s}^{*}\right)=1-\alpha$, from which we obtain the exponents

$$
\tau_{s}=2-1 / \lambda_{s}, \quad \tau_{s}^{*}=2-(1-\alpha) / \lambda_{s} .
$$

Using the previous relations, we can express Eq. (12) as a function of the exponents $\alpha$ and $\lambda_{s}$ alone:

$$
\left\langle s^{q}\right\rangle_{\theta}=\theta^{\lambda_{s}(q-1)+1}\left[C(q)+C^{*}(q) \theta^{-\alpha}\right] .
$$

Eq. (15) suggests the correct strategy to work out the moment analysis. Firstly, we observe that the quantity

$$
\Gamma_{q}(\theta) \equiv \frac{\left\langle s^{q}\right\rangle_{\theta}}{\theta^{\lambda_{s}(q-1)+1}}=C(q)+C^{*}(q) \theta^{-\alpha}
$$

depends only on $\theta^{-\alpha}$. We can use this fact to verify the validity of the ansatz $\lambda_{s}^{*}=\lambda_{s}$ by plotting $\Gamma_{q}(\theta)$ as a function of $\theta^{-\alpha}$ for different values of $q$, and checking whether or not the plots have linear dependence. Secondly, we 


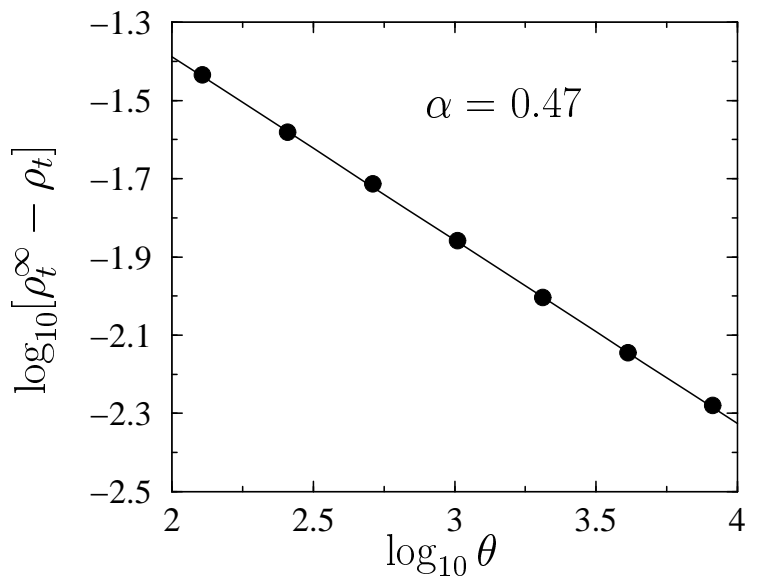

FIG. 1. Scaling of the average density of trees as a function of the parameter $\theta=p / f$.

note that the second term between brackets in the r.h.s. of (15) decreases with increasing $\theta$. For $\theta$ sufficiently large, this second term is negligible with respect to the constant $C(q),\left\langle s^{q}\right\rangle_{\theta}$ has a pure power-law dependence and we can proceed to compute $\lambda_{s}$ by means of linear regressions. Indeed, this observation allows us to define quantitatively the scaling region of the model: assuming that the ratio $C(q) / C^{*}(q)$ does not depend strongly on $q$, we define $\theta_{\text {scal }}$ as the value of the scaling parameter for which

$$
\frac{C^{*}(1)}{C(1)} \theta_{\text {scal }}^{-\alpha} \leq r,
$$

with $r$ some (arbitrary) small number. For $\theta>\theta_{\text {scal }}$, the approximation $\left\langle s^{q}\right\rangle_{\theta} \simeq \theta^{\lambda_{s}(q-1)+1} C(q)$, and therefore the single scaling form (1) is correct, within a precision of order $r$. One can thus proceed to compute the quantity $\sigma_{s}(q)$ by means of regressions limited to values of $\theta>$ $\theta_{\text {scal }}$, determine $\lambda_{s}$ by differentiation and, using Eq. (14), estimate the rest of the exponents.

\section{NUMERICAL RESULTS}

In order to check numerically our arguments, we have performed extensive numerical simulations of the FFM in $d=2$, using the algorithm described in Ref. [8]. Starting from an arbitrary initial configuration, we update the lattice according to the following rules: (i) select at random a site in the lattice; if the site contains a tree, burn it and all the trees that belong to its same forest cluster; if the site is empty, proceed to step ii; (ii) select at random $\theta$ sites; if a site is empty, grow a tree on it; if it contains a tree, do nothing. It is easy to see that these set of rules are equivalent to the original definition of the FFM, in the limit $p=0^{+}$and finite $p / f=\theta$. For large $\theta$, we thus ensure the double infinite time scale separation condition. The system sizes considered are up to $L=19000$

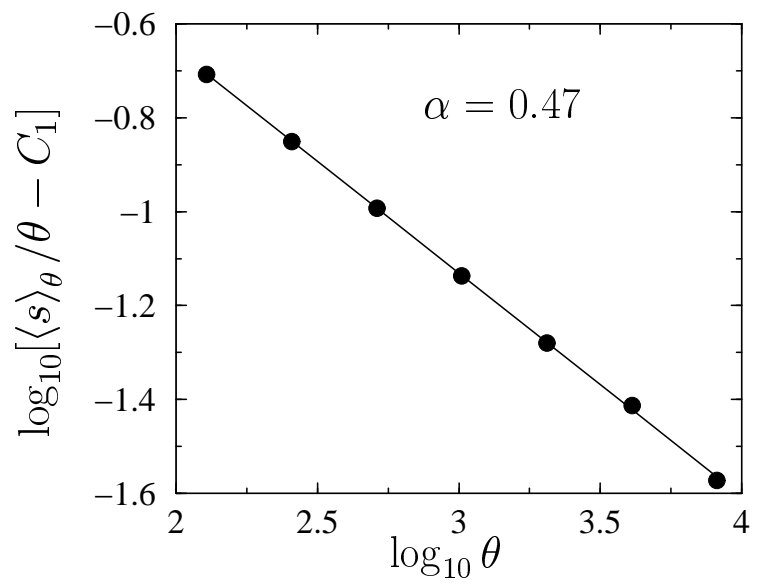

FIG. 2. Scaling of the average fire size as a function of the parameter $\theta=p / f$.

and the values of $\theta$ range from 128 to 32768 . Results are averaged over $10^{7}$ nonzero fires.

\section{A. Average density of trees}

In the first place, we study the average density of trees as a function of the parameter $\theta$. After discarding a sufficiently large number of fires (usually $5 \times 10^{5}$ ) to ensure that the system is in a steady-state, we compute the average number of trees, per unit area, left after each fire. The measured $\rho_{t}$ is fitted to the functional form $\rho_{t}^{\infty}-a \theta^{-\alpha}$ using the Levenberg-Marquardt non-linear fitting algorithm [19]. We obtain a critical asymptotic density of trees $\rho_{t}^{\infty}=0.4084 \pm 0.0005$, and an exponent $\alpha=0.47 \pm 0.01$, in good agreement with previous results [6]. In Figure 1 we have checked the asymptotic form of the average tree density by plotting $\log _{10}\left(\rho_{t}^{\infty}-\rho_{t}\right)$ as a function of $\log _{10} \theta$.

In Fig. 2 we check the validity of Eq. (6). The parameters computed, using again a non-linear curve fitting, are $C_{1} \simeq 0.854, C_{2} \simeq 1.973$, and $\alpha=0.47 \pm 0.01$. Again, we observe a very good fit to the predicted form. In view of this results, we select the value $\alpha=0.47$ for the computations to follow.

\section{B. Size probability distribution}

Once we have verified the likelihood of corrections to scaling in the first moment of the fire size distribution, we proceed to analyze the size probability distribution. The first step it to compute the threshold $\theta_{\text {scal }}$ using the criterion (17). We arbitrarily fix the parameter $r=0.05$; for this value, together with the estimates of $C_{1}=C(1)$ and $C_{2}=C^{*}(1)$ obtained by analyzing $\langle s\rangle_{\theta}$, we estimate $\theta_{\text {scal }} \geq 3000$. For values of $\theta$ larger than 3000 ; therefore, the single FSS form (11) can be assumed to be valid, 


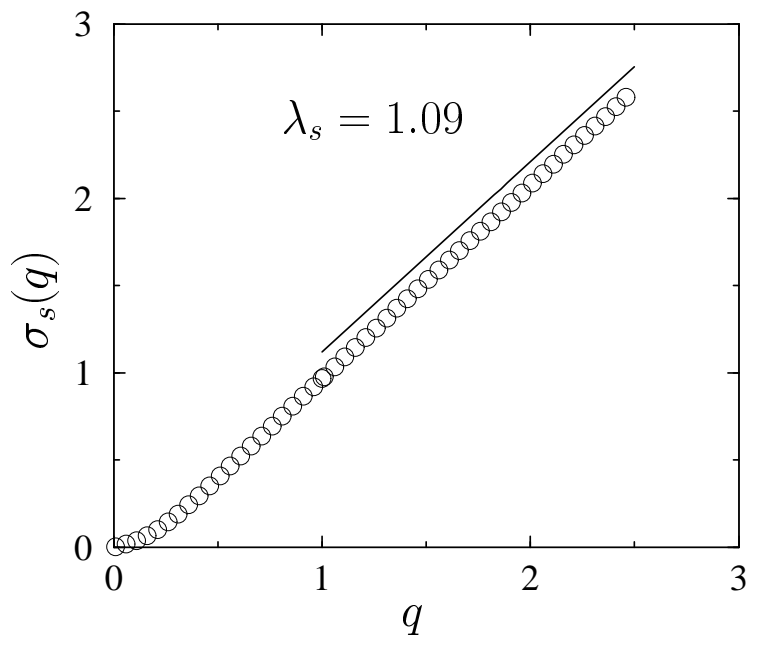

FIG. 3. Plot of $\sigma_{s}(q)$, computed from linear regressions from $\theta=4096$ to 32768 . The slope yields the exponent $\lambda_{s}=1.09 \pm 0.01$.

and we can proceed along the standard moment analysis technique. In Fig. 3 we plot the moments $\sigma_{s}(q)$ computed from linear regressions of $\log _{10}\left\langle s^{q}\right\rangle_{\theta}$ as a function of $\log _{10} \theta$, for values of $\theta$ between 4096 and 32768. The slope of this plot yields the exponent $\lambda_{s}=1.09 \pm 0.01$; finally using the relations (14) with $\alpha=0.47$, we obtain $\tau_{s}=1.08 \pm 0.01$ and $\tau_{s}^{*}=1.51 \pm 0.02$. A summary of results is presented in Table 1 .

Once we have computed the exponent $\lambda_{s}$, we can check a posteriori the validity of the ansatz $\lambda_{s}^{*}=\lambda_{s}$. We do so by plotting the quantity $\Gamma_{q}(\theta) / \Gamma_{q}\left(\theta_{\min }\right) \sim$ $\left\langle s^{q}\right\rangle_{\theta} / \theta^{\lambda_{s}(q-1)+1}$ as a function of $\theta^{-\alpha}$, with $\alpha=0.47$, for several values of $q$, Fig. 田. For large values of $\theta^{-\alpha}$, we observe a very good linear relationship. The goodness of the fit decreases for large $q$ and large $\theta$ (small $\theta^{-\alpha}$ ) because in both cases, the $q$-th moment is dominated by the largest avalanches, of which there is poorer statistics. We conclude therefore that indeed the assumption $\lambda_{s}^{*}=\lambda_{s}$ is indeed well justified for the FFM.

The presence of corrections of the form (7) make impossible to use a standard data collapse to inspect the accuracy of our results in the whole range of $\theta$ values. However, for $\theta>\theta_{\text {scal }}$, is reasonable to expect a good collapse to the single form (11). We have plotted this data collapse in Figure 5 , for the integrated size distributions.

\begin{tabular}{lccccc}
\hline \hline & $\tau_{s}$ & $\lambda_{s}=\lambda_{s}^{*}$ & $\tau_{s}^{*}$ & $\tau_{t}$ & $\lambda_{t}$ \\
\hline Slope & & & & & \\
Analysis & $1.14(3)$ & $1.15(3)$ & - & $1.27(7)$ & 0.58 \\
Moment & & & & & \\
Analysis & $1.08(1)$ & $1.09(1)$ & $1.51(2)$ & $1.27(1)$ & $0.59(1)$ \\
\hline \hline
\end{tabular}

TABLE I. Critical exponents for the FFM model, obtained through the slope analysis, Ref. [6], and by means of the moment analysis. Figures in parenthesis denote statistical uncertainties.

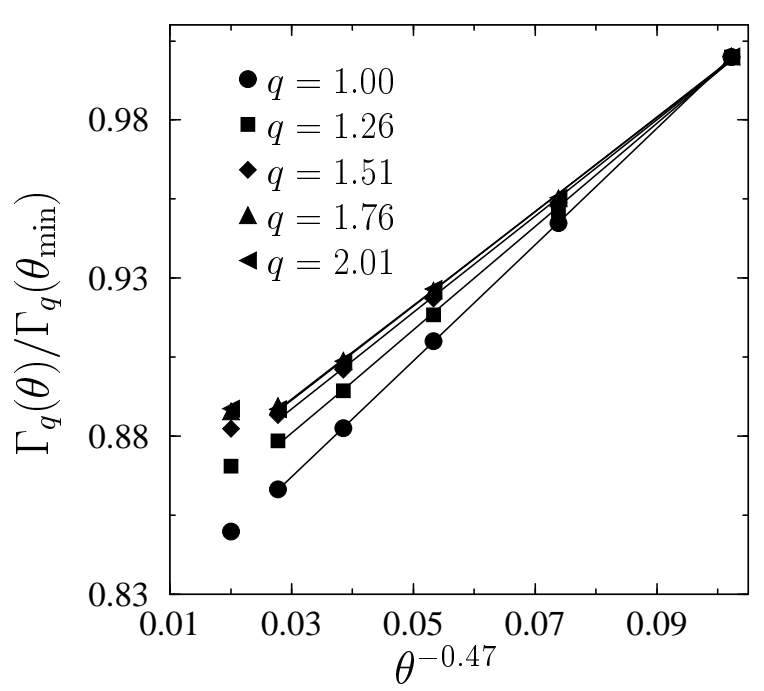

FIG. 4. Rescaled $q$-th moment $\Gamma_{q}(\theta) / \Gamma_{q}\left(\theta_{\min }\right)$ as function of $\theta^{-0.47}$. The good linear fit for small $\theta$ validates the ansatz $\lambda_{s}^{*}=\lambda_{s}$. The full lines are guides to the eye.

The collapse for the exponential tail of the distribution is quite remarkable. On the other hand, it is poorer for small values of $s$. This effect is due to the very presence of corrections to scaling, whose influence is stronger for small $s$.

Our method provides values which correct previous estimates (namely, in our notation, $\tau_{s}=1.14 \pm 0.03$ and $\lambda_{s}=1.15 \pm 0.03$, Ref. [6]) by a $5 \%$. As explained before, the discrepancy is due to the fact that in Ref. [6] exponents were computed by directly measuring the slope of the probability distributions, a method which is usually less accurate. Our value of $\lambda_{s}$, on the other hand, agrees better with the result reported in Ref. [8], which was obtained by a method closer in spirit to the moment analysis.

\section{Time probability distribution}

To complete the study of the FFM, we proceed in this section to apply the moment analysis to the fire time distribution. Here there is no a priori clue about the possible existence of corrections to scaling. We will therefore assume the simple FSS form (2) and perform the analysis for values of $\theta$ larger than $\theta_{\text {scal }}$.

Along the same lines followed for the size distribution, we define the $q$-th time moment $\left\langle t^{q}\right\rangle_{\theta}=\int_{1}^{\infty} t^{q} P(t, \theta) d t$. In this case, we have $\left\langle t^{q}\right\rangle_{\theta} \sim \theta^{\sigma_{t}(q)}$, with $\lambda_{t}=\partial \sigma_{t}(q) / \partial q$ and $\tau_{t}$ given by the relation $\left(2-\tau_{t}\right) \lambda_{t}=\sigma_{t}(1)$. In Figure 6 we plot $\sigma_{t}(q)$ as a function of $q$, computed by linear regression for the largest values of $\theta$. From the slope of this plot we obtain $\lambda_{t}=0.59 \pm 0.01$, and using this value on the precedent scaling relation, we obtain $\tau_{t}=1.27 \pm 0.01$. The data collapse with these exponents of the integrated time distribution is shown in Figure 7. In this case, and 


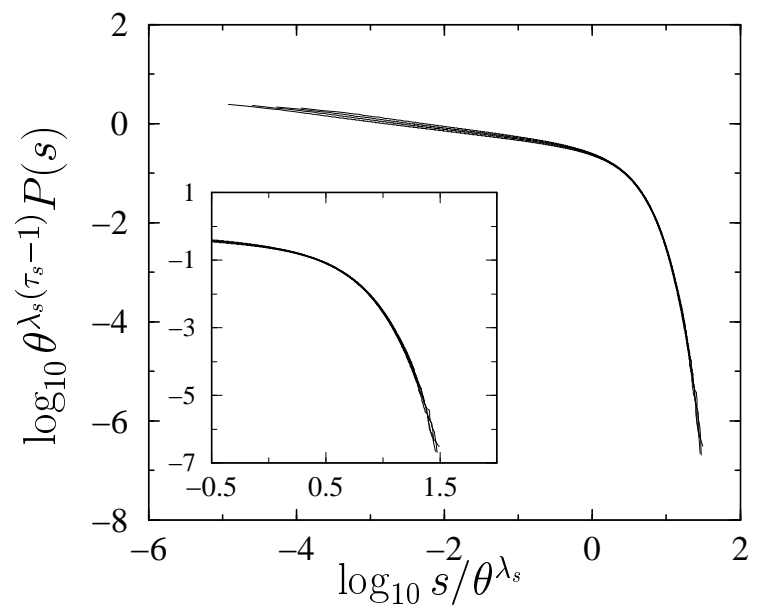

FIG. 5. Data collapse analysis of the integrated fire size distribution. $\theta=4096,8192,16384$, and 32768. Exponents used: $\lambda_{s}=1.09, \tau_{s}=1.08$. Inset: detail of the tail of the distribution.

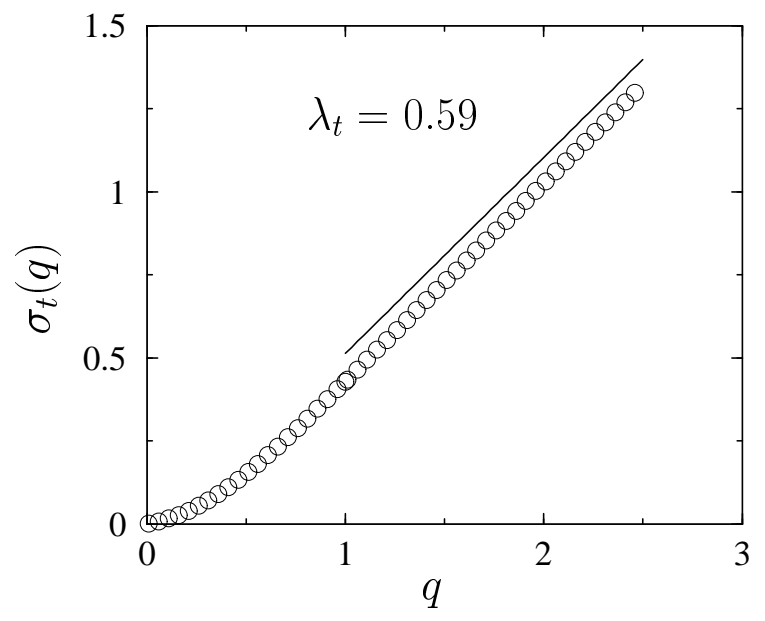

FIG. 6. Plot of $\sigma_{t}(q)$, computed from linear regressions from $\theta=4096$ to 32768 . The slope yields the exponent $\lambda_{t}=0.59 \pm 0.01$

on the contrary to the size distribution, the collapse is perfect for all values of $t$, which proves the irrelevance of corrections to scaling in the distribution of this magnitude.

It is interesting to note that our results match quite closely the results in [6], namely $\lambda_{t}=0.58$ and $\tau_{t}=$ $1.27 \pm 0.07$. This fact is accounted for by the method employed in [6] to compute $\lambda_{t}$, that is, an analysis of the lifetime of the largest fire, $T_{\max }$, as a function of $\theta$. This procedure indeed amounts to an estimation of the cut-off exponent of the time distribution, and is presumably less error-prone that a direct measurement of the initial slope of the distribution.

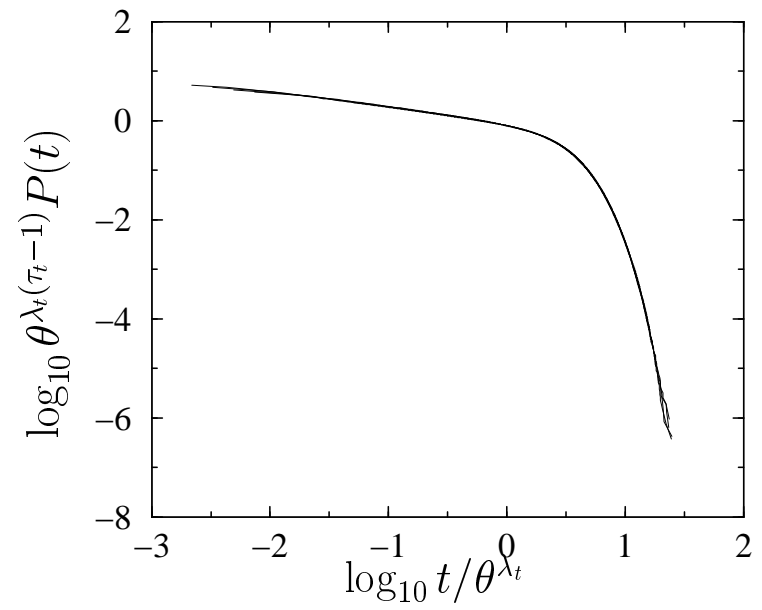

FIG. 7. Data collapse analysis of the integrated fire time distribution. $\theta=4096,8192,16384$, and 32768. Exponents used: $\lambda_{t}=0.59, \tau_{t}=1.27$.

\section{CONCLUSIONS}

In summary, in this paper we have shown that subdominant scaling corrections are inescapable in the forest-fire model. The analytical analysis of the stationarity condition shows that scaling corrections to a simple FSS form of the fires distribution must be included in order to account for the model behavior at finite values of $\theta$. In this perspective, we have proposed a method to explore corrections to the finite-size scaling hypothesis in the forestfire model. The method, based in an extension of the moment analysis, allows in principle the determination of the scaling regime of the models, as well as the computation of the first order corrections to scaling. Applying our method, we have been able to compute numerically corrected values to the scaling exponents, summarized in Table I, and estimate the first nontrivial corrections. We note that our approach is complementary with previous studies of deviation from scaling due to finite-size effects (small $L$ compared with $\theta$ ) 20.

As a final remark, it is interesting to point out that the present method can also be applied to standard sandpile models, defined on a lattice of size $L$ with open boundary conditions. In this case, however, the applicability of the method is hindered by the availability of a smaller range of values of the scaling parameter $L$. Interestingly, preliminar results with medium system sizes indicate that the ansatz $\lambda_{s}=\lambda_{s}^{*}$ may be violated in sandpiles. This fact can be related to the more complex structure of avalanches in sandpiles (compared with the percolationlike fires in the FFM), that induce the presence of more than one characteristic avalanche size. Unfortunately, the violation of the ansatz renders the computation of the corrections considerably much harder. Work is underways to explore the full structure of the corrections to scaling in sadpiles. 


\section{ACKNOWLEDGEMENTS}

We thank A. Stella for helpful discussions. This work has been supported by the European Network under Contract No. ERBFMRXCT980183.

[1] H. J. Jensen, Self-Organized Criticality (Cambridge University Press, Cambridge, 1998).

[2] P. Bak, C. Tang, and K. Wiesenfeld, Phys. Rev. Lett. 59, 381 (1987).

[3] P. Bak, K. Chen, and C. Tang, Phys. Lett. A 147, 297 (1992).

[4] B. Drossel and F. Schwabl, Phys. Rev. Lett. 69, 1629 (1992).

[5] A. Johansen, Physica D 78, 186 (1994).

[6] S. Clar, B. Drossel, and F. Schwabl, Phys. Rev. E 50, 1009 (1994).

[7] Finite Size Scaling, Vol. 2 of Current Physics-Sources and Comments, edited by J. L. Cardy (North Holland, Amsterdam, 1988).

[8] P. Grassberger, J. Phys. A: Math. Gen. 26, 2081 (1993).

[9] We have chosen a notation more conventional in sandpile models. The equivalence with the notation in Refs. [4, 6, 8], based on percolation theory, is $\tau_{s} \rightarrow \tau-1$, $\lambda_{s} \rightarrow \lambda, \tau_{t} \rightarrow b$, and $\lambda_{t} \rightarrow \nu^{\prime}$

[10] M. De Menech, A. L. Stella, and C. Tebaldi, Phys. Rev. E 58, R2677 (1998).

[11] C. Tebaldi, M. De Menech, and A. L. Stella, Phys. Rev. Lett 83, 3952 (1999).

[12] J. Cardy, Scaling and Renormalization in Statistical Physics (Cambridge University Press, Cambridge, 1996).

[13] S. Clar, B. Drossel, and F. Schwabl, J. Phys.: Condensed Matter 8, 6803 (1996).

[14] K. Christensen, H. Flyvbjerg, and Z. Olami, Phys. Rev. Lett. 71, 2737 (1993).

[15] A. Chessa, H. E. Stanley, A. Vespignani, and S. Zapperi, Phys. Rev. E 59, R12 (1999).

[16] A. Chessa, A. Vespignani, and S. Zapperi, Comput. Phys. Commun. 121-122, 299 (1999).

[17] R. Pastor-Satorras and A. Vespignani, J. Phys. A: Math. Gen. 33, L33 (2000).

[18] S. Lübeck, Phys. Rev. E 61, 204 (2000).

[19] W. H. Press, B. P. Flannery, S. A. Teukolsky, and W. T. Vetterling, Numerical Recipes in C: The Art of Scientific Computing, 2nd ed. (Cambridge University Press, Cambridge, 1992).

[20] K. Schenk, B. Drossel, S. Clar, and F. Schwabl, condmat/9904356. 